ARTICLE

\section{A Particle Consistent with the Higgs Boson Observed with the ATLAS Detector at the Large Hadron Collider}

\author{
The ATLAS Collaboration* $\dagger$
}

Nearly 50 years ago, theoretical physicists proposed that a field permeates the universe and gives energy to the vacuum. This field was required to explain why some, but not all, fundamental particles have mass. Numerous precision measurements during recent decades have provided indirect support for the existence of this field, but one crucial prediction of this theory has remained unconfirmed despite 30 years of experimental searches: the existence of a massive particle, the standard model Higgs boson. The ATLAS experiment at the Large Hadron Collider at CERN has now observed the production of a new particle with a mass of 126 giga-electron volts and decay signatures consistent with those expected for the Higgs particle. This result is strong support for the standard model of particle physics, including the presence of this vacuum field. The existence and properties of the newly discovered particle may also have consequences beyond the standard model itself.

$\mathrm{T}$ he standard model (SM) of particle physics (1-4) describes the fundamental particles and the electromagnetic, weak, and strong forces between them. It has been extremely successful at describing experimental data and predicting new results since its proposal in the 1960s. In the SM, forces are mediated by the exchange of particles with spin, which are known as bosons. These bosons are exchanged between electromagnetic, weak, and strong charges. The charges are carried by the fundamental constituents of matter: six quarks and six leptons, and their antiparticles, together known as fermions. The photon $(\gamma)$, the boson that mediates electromagnetism, and gluons, the bosons that mediate the strong force, are massless. However, the carriers of the weak force, the $\mathrm{W}$ and $\mathrm{Z}$ bosons - which are responsible for, for example, radioactivity and hydrogen fusion in the Sun - are observed to have masses $\sim 100$ times that of the proton. In the SM, the $\mathrm{W}$ and $\mathrm{Z}$ bosons obtain their mass through their interactions with a field of weak charge that is postulated to pervade the vacuum $(5-10)$ of space.

A critical prediction of the SM is that if enough energy is available, excitation of this vacuum field will produce a massive particle with zero spin: the Higgs boson, commonly denoted $\mathrm{H}$. The Higgs boson is fleeting; it is predicted to decay rapidly to other particles. Although many searches for the Higgs boson have been carried out since its prediction, it has remained elusive. The mass of the Higgs boson, $m_{\mathrm{H}}$, is not specified by the SM. Quantum mechanical effects link $m_{\mathrm{H}}$ to properties of known particles such as the W

*To whom correspondence should be addressed. E-mail: atlas. publications@cern.ch

†The complete author list is included as supplementary material on Science Online. boson and the t quark; the results of decades of precision measurements of such properties (11-13) indicate that $m_{\mathrm{H}}$ is $94_{-24}^{+29} \mathrm{GeV}$, but only at the $68 \%$ confidence level (CL) (14). This is slightly above the masses of the $\mathrm{W}$ and $\mathrm{Z}$ bosons. About 10 years ago, searches at the CERN Large Electron Positron (LEP) collider indicated that $m_{\mathrm{H}}$ was greater than $114.4 \mathrm{GeV}$ at the $95 \% \mathrm{CL}$ (15). After 25 years of collecting data, experiments at the Tevatron proton-antiproton collider at Fermilab recently excluded the mass region 147 to 180 $\mathrm{GeV}$ at the $95 \% \mathrm{CL}$ (10).

One of the main goals of the Large Hadron Collider (LHC) (17) physics program is to test this critical prediction of the SM: to either observe the SM Higgs boson and measure its properties or disprove its existence. The LHC accelerates two counter-rotating proton beams to nearly the speed of light so that the energy upon their collision should be sufficient to produce Higgs bosons over their expected mass range. The challenge is resolving the rare signal of the Higgs boson among a huge background of similar particles produced by the energetic collisions. Detection of a Higgs boson requires computing its mass from the total energy and momentum of all its decay particles. Unstable particles, such as $\mathrm{W}$ and $\mathrm{Z}$ bosons, may also be produced as intermediate quantum states with invariant masses well below their nominal masses.

Guided by our knowledge of the detector response to particles, we selected samples of events that the SM predicts to be enriched with Higgs bosons from the various decay channels. A Higgs boson with a mass of $\sim 126 \mathrm{GeV}$ would have five main experimentally accessible decay channels $(\mathrm{H} \rightarrow \gamma \gamma, \mathrm{ZZ}, \mathrm{WW}, \mathrm{bb}$, or $\tau \tau)$, where $\mathrm{b}$ denotes $\mathrm{a} \mathrm{b}$ quark, and $\tau$ denotes the heaviest lepton (tau). Evidence for Higgs boson production is inferred Other SM processes contribute to the background. from statistically significant excesses of events above the background predictions.

The LHC includes two detectors specifically designed for this search. In 2011, the LHC operated with a total proton-proton collision energy of $7 \mathrm{TeV}$. Both the A Toroidal LHC Apparatus (ATLAS) Collaboaration (18) and the Compact Muon Solenoid (CMS) Collaboration experiments (19) ruled out SM Higgs boson production in most of the remaining mass region considered relevant, from 110 to $600 \mathrm{GeV}$. However, the two experiments observed tantalizing hints of a new particle with mass in the region 124 to $126 \mathrm{GeV}$ and compatible with a SM Higgs boson $(20,21)$.

The LHC's collision energy was raised to 8 $\mathrm{TeV}$ in 2012, increasing the predicted production rate of SM Higgs bosons and the sensitivity of the search. In order to avoid observer bias, all details of the analyses, such as the set of search channels, the event selection criteria, and the signal and background predictions, were fixed before examining the signal regions of the April-June 2012 data. Here, we report the discovery of a SM Higgs-like boson in the combined data collected with the ATLAS detector during 2011 and AprilJune 2012. This paper provides an overview of the experimental results that are described in more detail in (22). Independently, the CMS experiment also identified a similar boson at the same mass, as discussed in $(23,24)$.

The ATLAS detector. The design of the cylindrically symmetric ATLAS detector (18) was optimized to study a broad range of physics processes, including SM Higgs boson production, over a wide mass range. The entire detector (Fig. 1) weighs 7000 metric tons. It is $44 \mathrm{~m}$ long and $25 \mathrm{~m}$ in diameter. It is located in an underground cavern at a depth of $100 \mathrm{~m}$, where it surrounds one of the collision points around the $27-\mathrm{km}$-long LHC ring. ATLAS is actually composed of several distinct subdetectors in order to identify and measure the energy and momentum of a variety of particles and so reconstruct the dynamics of the collision.

The momenta of charged particles are measured by an inner tracking detector (ID) immersed in a 2-T axial field provided by a superconducting magnet. The energies of electrons and photons are measured in an electromagnetic calorimeter (ECAL) that surrounds the inner detector and magnet. An additional layer of calorimeters outside the ECAL for measuring hadrons (such as protons and neutrons) also serves as an absorber, so that only energetic muons and the elusive weakly interacting neutrinos penetrate it. The muon spectrometer surrounds the calorimeters; it consists of superconducting magnets providing a toroidal field and a system of precision charged-particle detectors.

The combination of the subdetectors provides particle energy and momentum measurements, together with electron, muon, and photon identification, over more than $98 \%$ of the solid angle. The measurements are made by $\sim 90$ million sensor elements, most of which are in the inner de- 
tector. Jets (narrow cones of particles produced by the conversion of quarks and gluons to hadrons) are reconstructed by using the nearly $4 \pi$ solid angle coverage of the calorimeters. In highenergy proton-proton collisions, only one constituent (a quark or a gluon) from each proton takes part in the interactions that result in a wide variety of known and possibly unknown processes, including production of the SM Higgs boson. The remnants of two colliding protons tend to travel along the beam directions and exit the detector unobserved, so it is only possible to study momentum balance in the plane transverse to the proton beam axis. Neutrinos, which are normally not directly detectable, are inferred from their transverse momenta; they are assumed to balance the sum of the transverse momenta of the observed electrons, muons, photons, and jets: Their presence is thus indicated by the magnitude of the missing transverse momentum, denoted $E_{\mathrm{T}}^{\text {miss }}$.

During standard LHC operation, two counterrotating packets of protons cross at the center of ATLAS every $50 \mathrm{~ns}$. The high intensity of the proton beams results in multiple proton-proton collisions occurring during each crossing of proton packets, an effect known as "pile-up" (Fig. 2). The average number of interactions per proton-packet crossing was $\sim 10$ in 2011; it increased to $\sim 20$ in 2012, but advances in understanding the detector performance and improved analysis techniques mitigate the effects of the harsher environment.

The set of digitized signals recording the collision products of a single crossing of proton pack- ets is known as an event. A three-level trigger system decides which events should be recorded; typically, 20 potentially interesting events are selected out of 1 million produced. Each trigger level reduces the rate by a factor between 10 and 100 . In this way, only the most interesting events (those with high transverse momentum electrons, muons, photons, or jets) are recognized and recorded. Each event requires $\sim 1$ megabyte of storage, and typically 400 events are recorded every second.

Further details of the design of the detector are given in (25).

Signal expectation and background estimation. The most important SM Higgs boson production process in the energy range of the LHC is expected to be gluon fusion. Gluons do not directly produce the SM Higgs boson but rather do so indirectly through a quantum loop process involving mainly the heaviest $(\mathrm{t})$ quark (Fig. 3). Other processes are predicted to provide much clearer signals but at substantially reduced rates. The production and decay rates used to infer signal yields in our analysis are taken from theoretical predictions $(26,27)$.

The background rates and signal efficiencies are estimated from the data as far as possible. To supplement this, we simulated the production of the SM Higgs boson signal and relevant background processes. These simulations use mathematical functions, constrained by experimental data, to describe the energy distributions of quarks and gluons in the colliding protons and how they interact and describe in detail how the outgoing particles behave in the ATLAS detector $(28,29)$. They also include modeling of the pile-up conditions observed in the data.

The SM predicts that $\sim 200,000$ SM Higgs bosons are produced in the combined ATLAS data if $m_{\mathrm{H}}$ is $126 \mathrm{GeV}$. However, because most of the decays are indistinguishable from the $8 \times 10^{14}$ inelastic proton-proton collisions in the combined ATLAS data, we focused on a few distinctive SM Higgs boson decay modes. Two of the most sensitive channels are the decay into two photons (denoted the $\mathrm{H} \rightarrow \gamma \gamma$ channel) and the decay into two $\mathrm{Z}$ bosons, which in turn each decay into an oppositely charged pair of electrons or muons [denoted the $\mathrm{H} \rightarrow \mathrm{ZZ} \rightarrow \ell \ell \ell \ell$ channel (30)]. Both of these channels were examined in the data from 2011 and 2012. An additional sensitive decay mode involving two $W$ bosons decaying to an electron, a muon, and two neutrinos (denoted the $\mathrm{H} \rightarrow \mathrm{WW} \rightarrow e v \mu \nu$ channel) was included in the 2012 search. Additional channels in which the SM Higgs boson decays to pairs of $b$ quarks or $\tau$ leptons, or alternative decay patterns for the $\mathrm{W}$ or $\mathrm{Z}$ bosons, have so far been studied in 2011 data only because it takes more time to study their more complex signatures.

$\mathbf{H} \rightarrow \boldsymbol{\gamma} \boldsymbol{\gamma}$ channel. The decay of the SM Higgs boson to a pair of photons proceeds mainly via quantum loop processes involving the $\mathrm{W}$ boson, as illustrated in Fig. 4. The fraction decaying in this way is never large, typically $0.2 \%$; however, the signature of two high-energy photons isolated from any other sizable activity in the

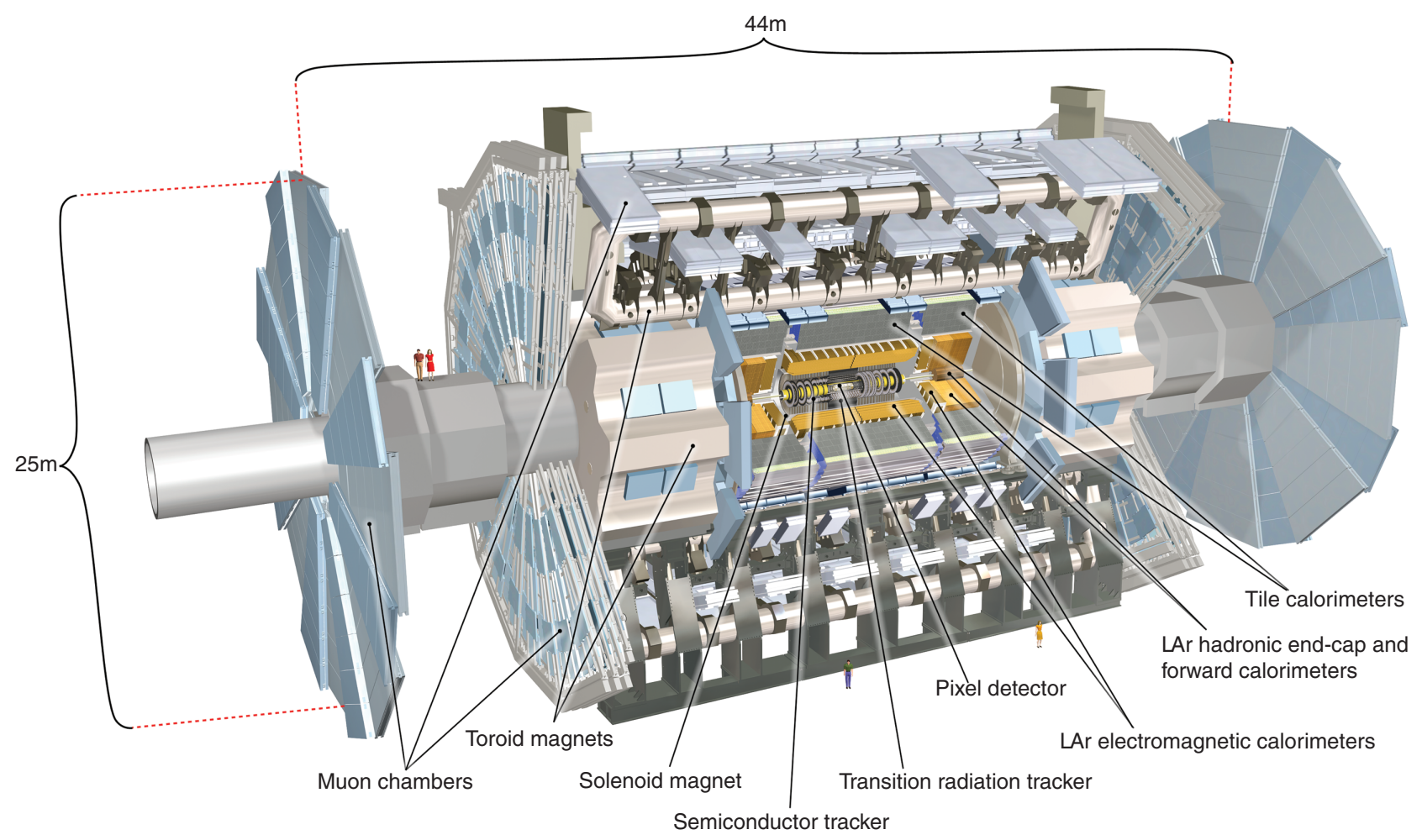

Fig. 1. Cutaway drawing of the ATLAS detector showing its main components. 


\section{The Higgs Boson}

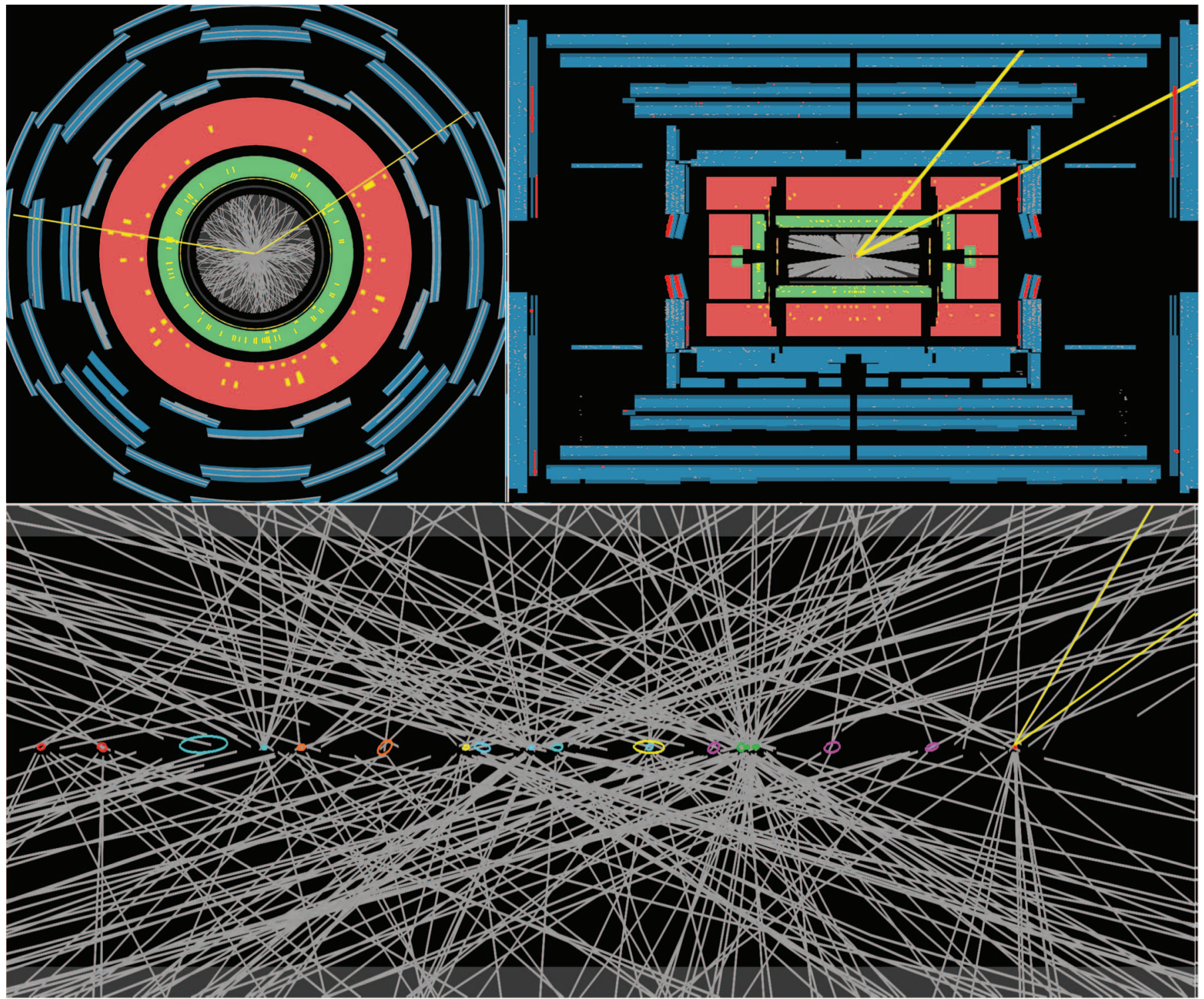

Fig. 2. A candidate $Z$ boson decay to $\mu^{+} \mu^{-}$with 20 reconstructed vertices (the typical pile-up condition in the $8 \mathrm{TeV}$ data). (Top) The transverse (left) and longitudinal (right) projections in the full ATLAS detector where the two muons (yellow) are clearly iden- tified. (Bottom) The detail of the 20-cm-long vertex region. The two muons can both be seen to emerge from the same vertex. The error ellipses of the reconstructed vertices are shown scaled up by a factor of 10 so that they are visible. detector is distinctive. Owing to the excellent mass measurement, this channel should show a narrow peak with width $\sim 1.3 \%$ in an otherwise featureless $\gamma \gamma$ mass spectrum of background in the search range between 110 and $150 \mathrm{GeV}$.

The signal-to-background ratio was improved with strict photon identification and other requirements; this reduced the enormous jet background by a factor of $\sim 10^{8}$ while keeping almost half of the predicted $\mathrm{H} \rightarrow \gamma \gamma$ signal events. The majority of the remaining background consists of genuine photon pairs from processes that do not involve a SM Higgs boson. A typical candidate for a $\mathrm{H} \rightarrow \gamma \gamma$ decay is shown in Fig. 5. Nearly all such events are from background processes.

The di-photon mass was calculated from the measured properties of the photons. The depth

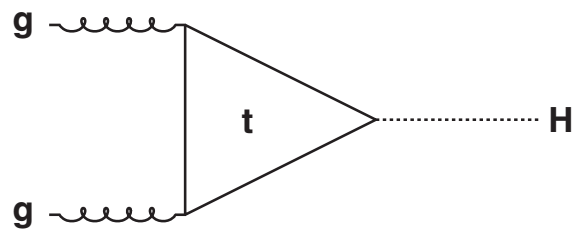

Fig. 3. Feynman diagram illustrating the dominant Higgs boson production mechanism at the elementary level at the LHC: production of a Higgs boson, $\mathrm{H}$, by gluon fusion and a quantum loop process involving a t quark.

segmentation of the calorimeter allows the directions of the photons to be measured. Extrapolating these back to the beam-line gives the position of the production position to an accuracy of $15 \mathrm{~mm}$,

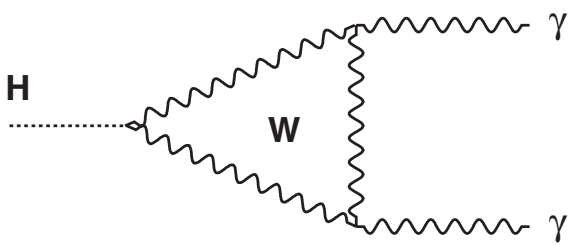

Fig. 4. Feynman diagram illustrating the decay mechanism of a Higgs boson, $\mathrm{H}$, to two photons, $\gamma$ via quantum loop process involving a $\mathrm{W}$ boson.

which is sufficient to precisely determine the mass None of the background types forms a sharp peak at any di-photon mass in the search region.

The predicted signal yield in the full set of 59,039 selected events was $\sim 190$ events for $m_{\mathrm{H}}=$ 


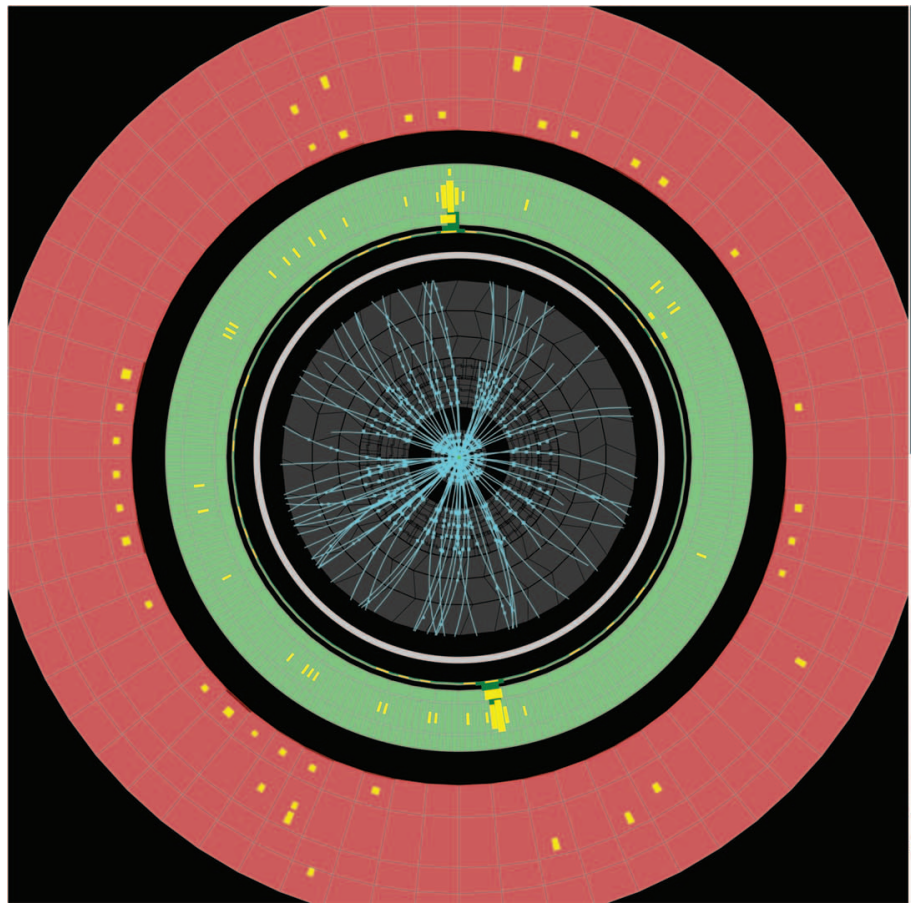

Fig. 5. Display of a $\mathrm{H} \rightarrow \gamma \gamma$ event candidate in the $8 \mathrm{TeV}$ data. Energy deposits are shown in yellow in the ECAL (green) and hadronic calorimeter (red). Tracks from charged particles and the associated space points measured by the ID are shown in blue. Views of the calorimeter systems and ID are shown along the proton-proton collision axis (top middle) and transverse to it (left). The bottom middle and bottom right panels show a magnified

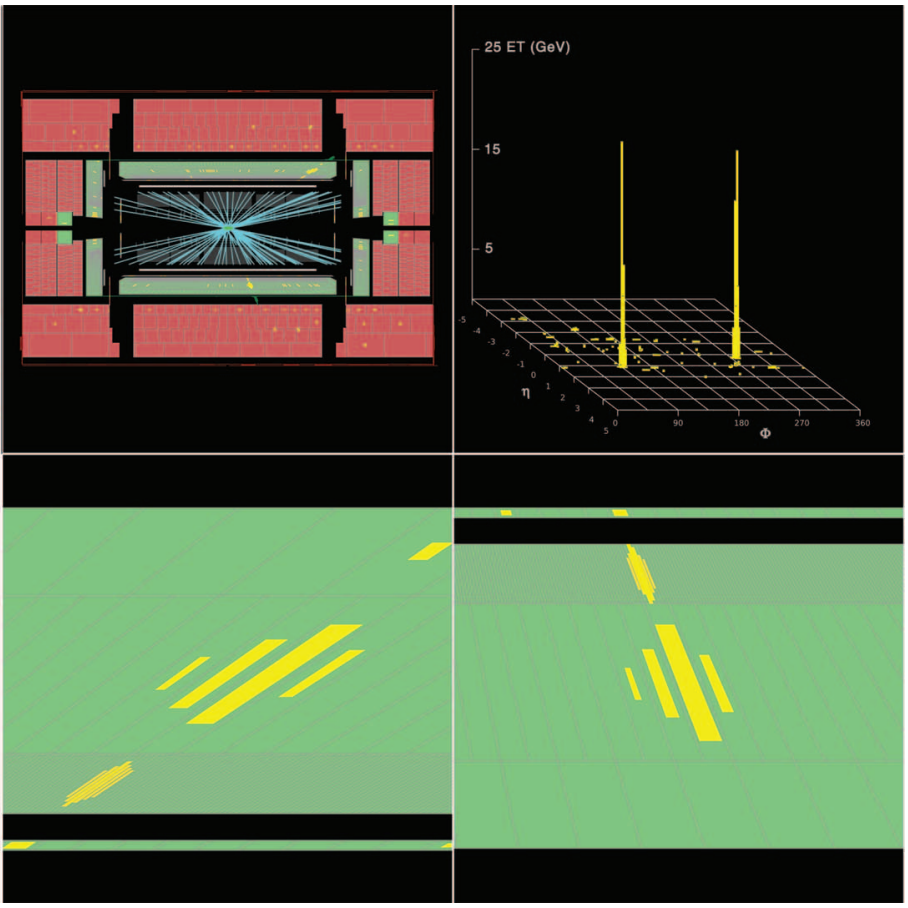

display of the response of the fine-grained ECAL in the longitudinal view for the two photon candidates. Photons are rapidly stopped in the dense ECAL, and these truncated showers match that expectation. The plot on the top right shows the energy depositions projected into azimuthal and longitudinal coordinates-unrolling the calorimeter. The measured mass of this photon-candidate pair is $126.9 \mathrm{GeV}$.
$126 \mathrm{GeV}$. We enhanced the sensitivity of the analysis by assigning the events to 10 mutually exclusive categories, each with different signal purities and mass resolutions. The signal was extracted by a global fit to the $\gamma \gamma$ mass spectra in the categories. Each spectrum is described by a smooth parametric background model plus a signal model, taken from simulation, which is approximately Gaussian but includes broader tails. For representational purposes, these 10 categories are combined into a single di-photon mass plot, Fig. 6, in which a small but statistically significant excess can be seen.

$\mathbf{H} \rightarrow \mathbf{Z Z} \rightarrow \boldsymbol{\ell \ell \ell \ell}$ channel. The search channel in which the SM Higgs boson decays to two $\mathrm{Z}$ bosons, each then decaying to either $e^{+} e^{-}$or $\mu^{+} \mu^{-}$, offers the best signal purity, over $50 \%$. However, because only $7 \%$ of $Z$ bosons decay like this, the rate is low: About six signal events were expected in our data sample for $m_{\mathrm{H}} \approx 126$ $\mathrm{GeV}$. Owing to the precise momentum and energy measurements of leptons, a SM Higgs boson with $m_{\mathrm{H}} \approx 126 \mathrm{GeV}$ will produce a narrow peak of width $\sim 1.5 \%$ in the measured four-lepton mass spectrum. The observed four-lepton mass spectrum is consistent with the predicted background, as seen in Fig. 7, apart from the narrow excess of events with mass of $\sim 126 \mathrm{GeV}$.

$\mathbf{H} \rightarrow \mathbf{W W} \rightarrow \boldsymbol{\ell} \boldsymbol{v} \boldsymbol{\ell} \boldsymbol{v}$ channel. The decay $\mathrm{H} \rightarrow \mathrm{WW} \rightarrow$ $\ell v \ell v$ also provides good sensitivity for $m_{\mathrm{H}}=$

Fig. 6. Distribution of the mass, $m_{\gamma \gamma}$ of weighted di-photon candidates. The selected events are weighted by factors that reflect the signal-tobackground ratio predicted for a SM Higgs boson. The result of a fit to the data of the sum of a signal component fixed to $m_{\mathrm{H}}=126.5 \mathrm{GeV}$ and $\mathrm{a}$ background component described by a fourthorder polynomial are superimposed. The residuals of the weighted data with respect to the fitted background are displayed at the bottom. Collision en-

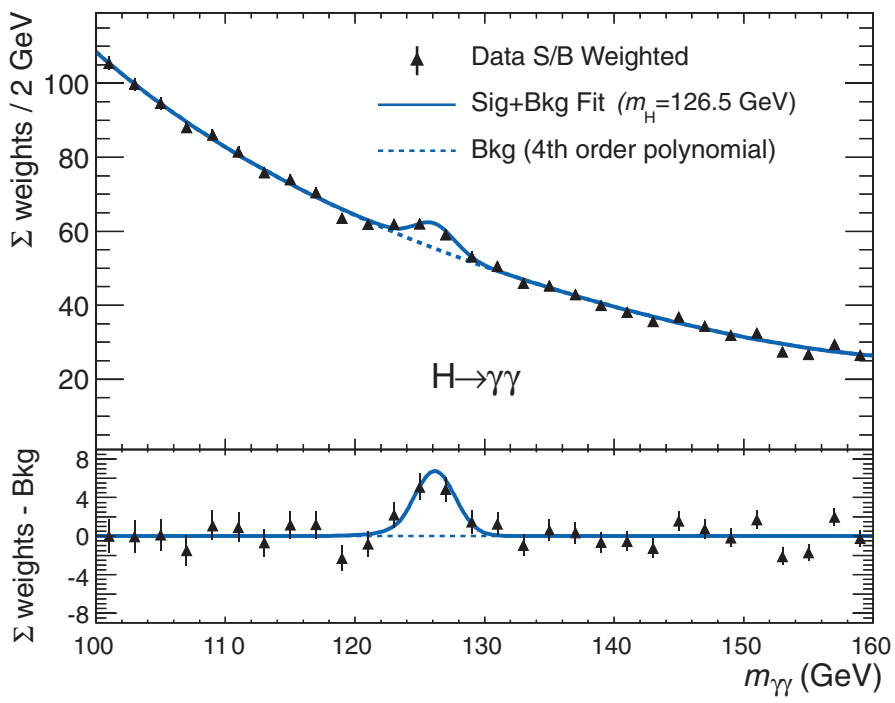
ergy $=7 \mathrm{TeV}$, integrated luminosity $(\mathrm{L})=4.8$ inverse femtobarns $\left(\mathrm{fb}^{-1}\right)$; collision energy $=8 \mathrm{TeV}, \mathrm{L}=5.9 \mathrm{fb}^{-1}$.

$126 \mathrm{GeV}$, owing to the relatively large predicted number of events combined with the purely leptonic signature. However, the presence of two undetectable neutrinos in the final state means that a full reconstruction of each event is impossible. Consequently, the masses of the Higgs boson candidates cannot be calculated. However, a "transverse mass," $m_{\mathrm{T}}$, which is sensitive to the
Higgs boson mass, was constructed from the detected leptons and missing transverse momentum (31). Because the presence of a signal is primarily inferred via a difference between the event rate observed and that expected from background only, and the expected signal yield is only about 15 to $20 \%$ of the background rate in the region of interest, the background rate and composition 
must be understood to a precision considerably better than $20 \%$. To accomplish this, we isolated all of the largest background components in kinematically nearby regions of data in which no Higgs boson signal is expected and extrapolated the measured background rates into the signal region. The largest background in the final selection was in fact from direct WW production, which does not involve a Higgs boson.

The $E_{\mathrm{T}}^{\text {miss }}$ resolution in the $8 \mathrm{TeV}$ data is degraded by increased pile-up, which increases the background rates, especially for high-mass pairs of leptons of the same type. To circumvent this, we decided to use only events with an electron-muon pair for the $8 \mathrm{TeV}$ data (one such event is shown in Fig. 8). The distribution of transverse mass for these events is shown in Fig. 9, in which $\sim 40$ signal events are predicted for $m_{\mathrm{H}}=126 \mathrm{GeV}$.

Statistical procedures. Because the SM makes a specific prediction for how the SM Higgs boson is produced and decays, the results in all production and decay channels and data sets are combined into a single likelihood function (20). The likelihood depends on a signal strength parameter, $\mu$, which is a scale factor on the total number of events predicted by the SM for a Higgs boson signal. It is defined so that $\mu=$ 0 corresponds to the background-only hypothe-

Fig. 7. The distribution of the mass of the selected $\mathrm{H} \rightarrow \mathrm{ZZ} \rightarrow \ell \ell \ell$ candidate events, $m_{\text {eece }}$. The small peak at 90 $\mathrm{GeV}$ corresponds to a single $\mathrm{Z}$ boson decaying to four leptons, whereas the broad structure around $200 \mathrm{GeV}$ results from the direct production of $Z$ boson pairs. An excess is seen around $125 \mathrm{GeV}$; the expected signal from a SM Higgs boson at that mass (light blue) is added for comparison. The hatched area indicates the systematic uncertainty in the background estimation. Collision energy $=7 \mathrm{TeV}, \mathrm{L}=$ $4.8 \mathrm{fb}^{-1}$; collision energy $=8 \mathrm{TeV}, \mathrm{L}=$ $5.8 \mathrm{fb}^{-1}$.

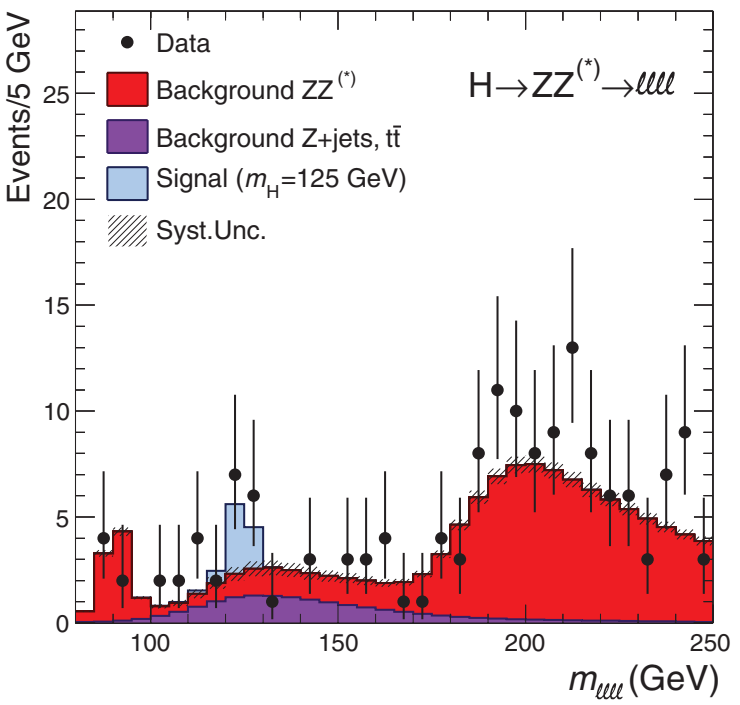

sis, and $\mu=1$ corresponds to the predicted Higgs boson signal in addition to the background.

The likelihood is calculated as the product of the probabilities of observing each event, where the individual event probabilities depend on the measured masses (or $m_{\mathrm{T}}$ ) of the Higgs boson candidates. The evaluation accounts for systematic uncertainties. The signal strength and the parameters that describe the systematic uncertainties are varied to maximize the likelihood of the model used to describe the observed data. The ratio of the likelihood with the best-fit signal to that with a specified signal, $\mu=1$ or 0 , is calculated; these likelihood ratios are then used to quantify the exclusion of the signal hypothesis or the rejection of the background hypothesis, respectively.

The statistical tests were repeated at various values of $m_{\mathrm{H}}$ and $\mu$. A SM Higgs boson with mass $m_{\mathrm{H}}$ was considered excluded when $\mu=1$ is excluded at $95 \% \mathrm{CL}$ at that mass. This is equivalent to the upper limit on $\mu$ at $95 \%$ CL being less than 1 . On the other hand, a significant rejection of the background hypothesis was interpreted as evidence for the SM Higgs boson because this is the alternate hypothesis. The significance is quantified with the local $P$ value, the probability that the background can randomly fluctuate to produce a measured likelihood ratio at least as signal-like as the excess observed in the data; it is also expressed in terms of the equivalent number of standard deviations of a normal distribution $(\sigma)$ and is then referred to as the local significance.

Because the SM does not predict the value of $m_{\mathrm{H}}$, and because background fluctuations can occur anywhere in the search region of $m_{\mathrm{H}}$, the

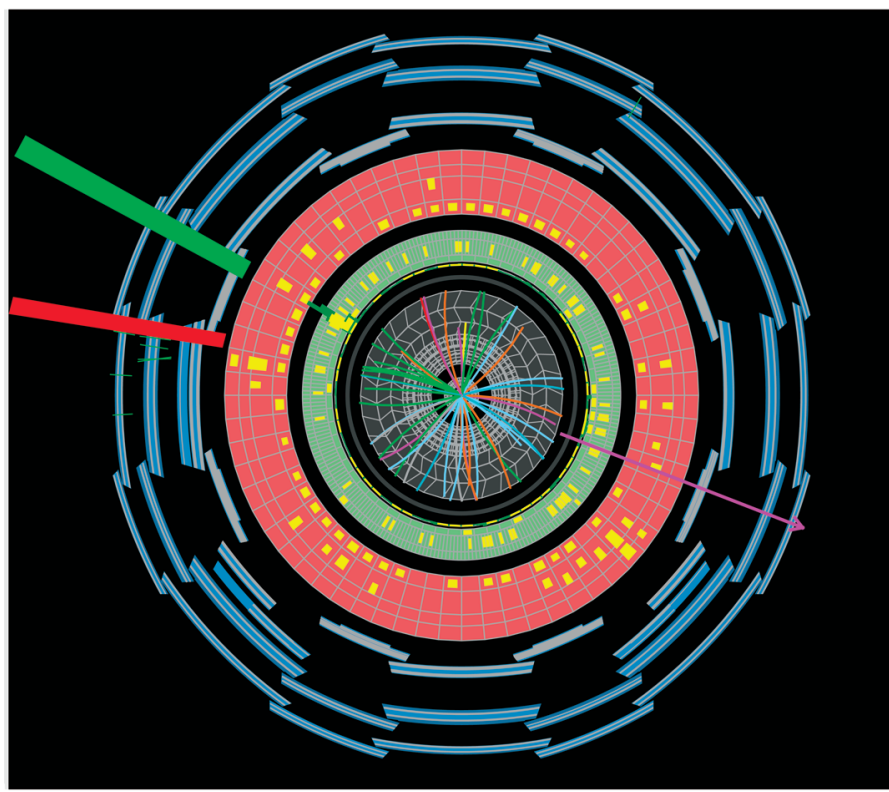

Fig. 8. A candidate event for $\mathrm{H} \rightarrow \mathrm{WW} \rightarrow$ ev $\mu \nu$ shown in transverse (left) and longitudinal (top right) projections through the complete detector. Under the SM Higgs boson hypothesis with $m_{\mathrm{H}}=126 \mathrm{GeV}, 90 \%$ of such events are background. The electron and muon directions are highlighted with green and red markers, respectively. They are on the opposite side of the detector from a
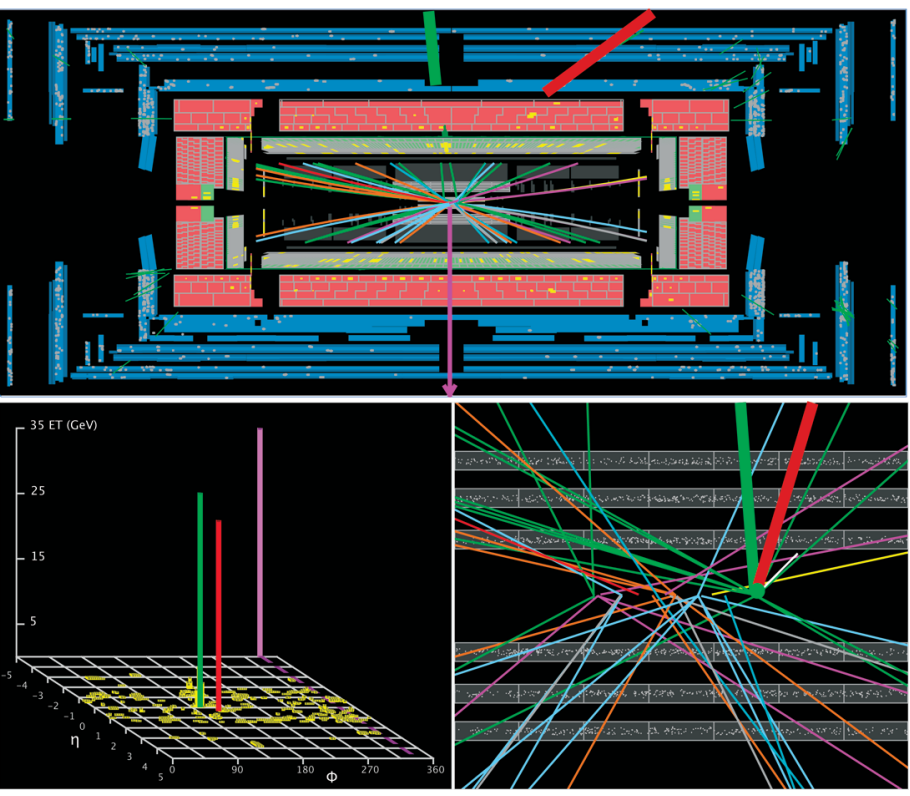

large amount of $E_{\mathrm{T}}^{\text {miss }}$ (magenta), and they both come from the same vertex (bottom right). The plot on the bottom middle shows the energy deposition projected into azimuthal and longitudinal coordinates; because $E_{\mathrm{T}}^{\text {miss }}$ is only defined in azimuth, the line representing it is arbitrarily placed at the farthest longitudinal position. 


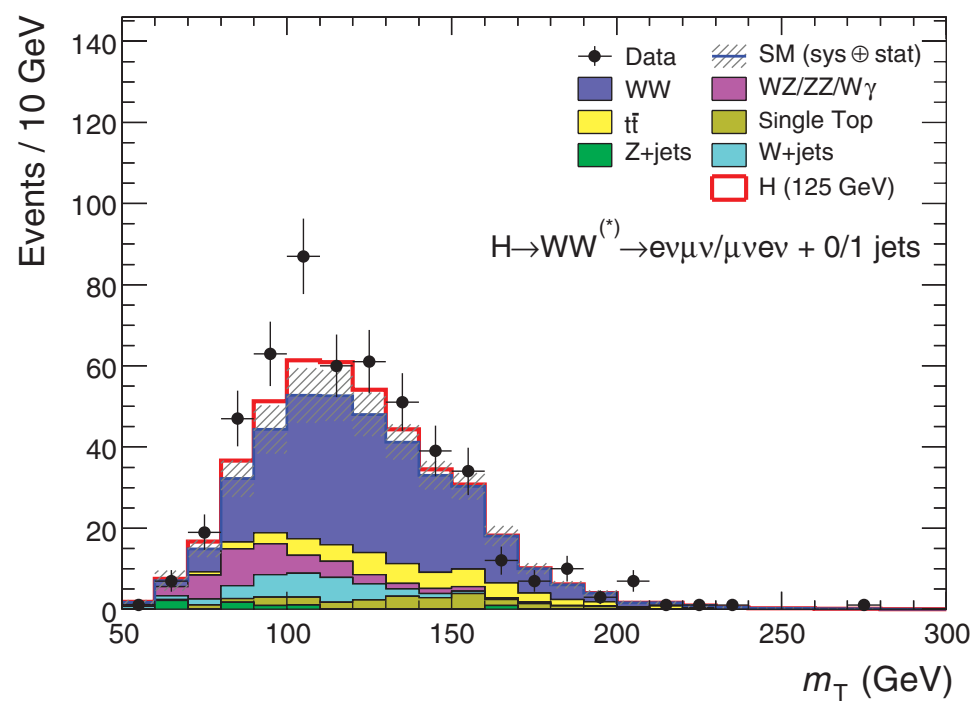

Fig. 9. The distribution of transverse mass, $m_{\mathrm{T}}$, for $\mathrm{H} \rightarrow \mathrm{WW} \rightarrow$ ev $\mu \nu$ candidates in the $8 \mathrm{TeV}$ data taken during April-June 2012. The red histogram indicates the predicted enhancement of the distribution for $m_{\mathrm{H}}=125 \mathrm{GeV}$. The hatched band indicates the total uncertainty on the background estimation. Collision energy $=8 \mathrm{TeV}, \mathrm{L}=5.8 \mathrm{fb}^{-1}$.

Fig. 10. Combined search results: the measured (solid) $95 \%$ CL upper limits on the signal strength as a function of $m_{\mathrm{H}}$ and the expectation (dashed) under the background-only hypothesis. The green and yellow bands show the $\pm 1 \sigma$ and $\pm 2 \sigma$ uncertainties on the background-only expectation, respectively. In the broad region where the expected limit is below the signal strength for the SM, $\mu=1$, there is sensitivity to exclude a Higgs boson in its absence. There are two regions of Higgs boson mass that are not excluded by

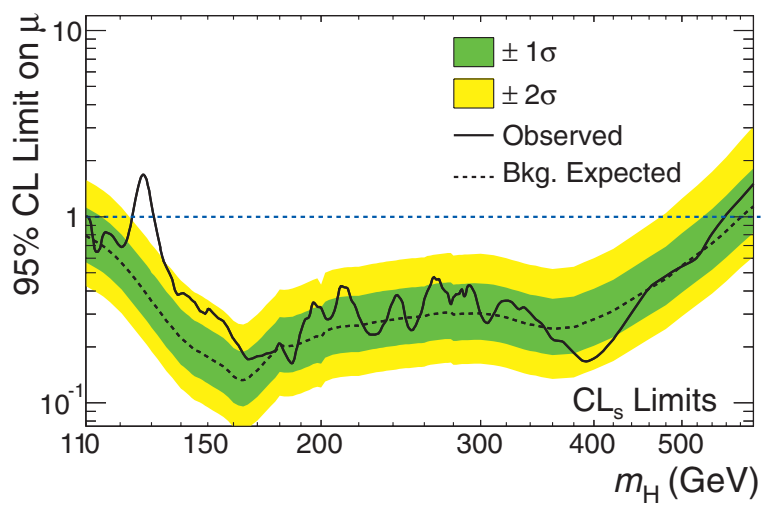
the data. There is a mild failure to exclude at high mass and a significant failure to exclude around $m_{\mathrm{H}}=$ $126 \mathrm{GeV}$. Collision energy $=7 \mathrm{TeV}, \mathrm{L}=4.6$ to $4.8 \mathrm{fb}^{-1}$; collision energy $=8 \mathrm{TeV}, \mathrm{L}=5.8$ to $5.9 \mathrm{fb}^{-1}$.

Fig. 11. Combined search results: the observed (solid) local $P$ as a function of $m_{\mathrm{H}}$ and the expectation (dashed) for a SM Higgs boson signal hypothesis $(\mu=1)$ at the given mass. The shaded band indicates the expected $\pm 1 \sigma$ variation of the signal prediction. Collision energy $=$ $7 \mathrm{TeV}, \mathrm{L}=4.6$ to $4.8 \mathrm{fb}^{-1}$; collision energy $=8 \mathrm{TeV}$, $\mathrm{L}=5.8$ to $5.9 \mathrm{fb}^{-1}$.

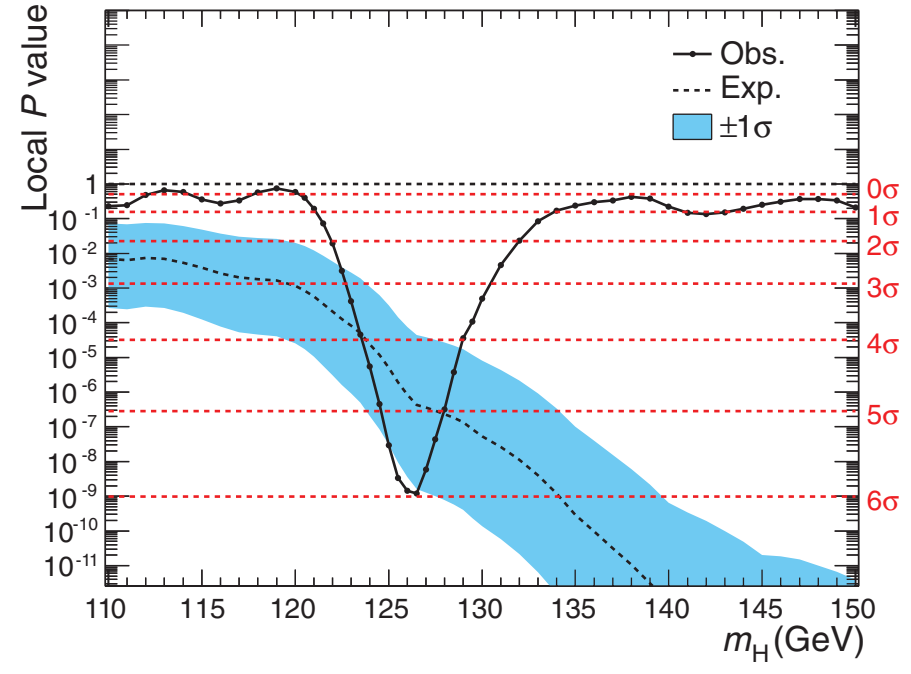

local significance is an overestimate of the true significance. The global significance corrects for this "look-elsewhere" effect.

Observation of a SM Higgs-like boson. The combined search used the full $7 \mathrm{TeV}$ data collected in 2011 and the $8 \mathrm{TeV}$ data from AprilJune 2012 in the most sensitive channels. The latter data provide considerable gains in sensitivity with respect to the search based on 2011 data only (20). In the absence of a signal, this should allow the exclusion of the SM Higgs boson for all masses between 110 and $582 \mathrm{GeV}$, as shown in Fig. 10. This range overlaps with the lower bound from LEP (114.4 GeV); if the entire range had been excluded, this would have shown the SM to be deeply flawed. Our data exclude a SM Higgs boson signal at 95\% CL in two mass regions, 111 to $122 \mathrm{GeV}$ and 131 to $559 \mathrm{GeV}$ (Fig. 10). In the region around $126 \mathrm{GeV}$, this analysis is more than sensitive enough to exclude a SM Higgs boson signal at $95 \% \mathrm{CL}$; the failure to do so means that the possibility of a discovery must be considered. Indeed, as shown in Figs. 6, 7, and 9, an excess of events is observed near $m_{\mathrm{H}}=126 \mathrm{GeV}$.

The largest local significance for the combined data is for a SM Higgs boson mass of $m_{\mathrm{H}}$ $\sim 126 \mathrm{GeV}$, at which it reaches $6.0 \sigma$, corresponding to a probability of an upward fluctuation of the background of $1.0 \times 10^{-9}$. This significance is slightly higher than, but consistent with, the expected SM Higgs boson signal at this mass, as seen in Fig. 11. The observed significances for the statistically independent 7 and $8 \mathrm{TeV}$ data samples both peak at $\sim 126 \mathrm{GeV}$, at which they are $3.6 \sigma$ and $4.9 \sigma$, respectively. Uncertainties in the relative energy scales of the detector for electrons and muons reduce the combined local significance to $5.9 \sigma$. The global significance of the excess is $\sim 5.1 \sigma$.

It is now crucial to establish how well this observation corresponds (or not) to the SM Higgs boson. The consistency of the production rates in the three primary channels with the predictions of the theory is confirmed with a simultaneous fit to $\mu$ and $m_{\mathrm{H}}$, as shown in Fig. 12, in which the CL contours take into account all systematic uncertainties, including the effects of the energy scale and resolution. The positions of the mass peaks in the two channels with the best mass resolution, $\mathrm{H} \rightarrow \gamma \gamma$ and $\mathrm{H} \rightarrow \mathrm{ZZ} \rightarrow \ell \ell \ell \ell$, are consistent with the observation of a single new particle. The measured mass of the observed particle is $126.0 \pm 0.4 \pm$ $0.4 \mathrm{GeV}$, where the two uncertainties are statistical and systematic, respectively. The leading sources of systematic uncertainty come from the photon and, to a lesser extent, electron energy scales.

The signal strength for the fitted mass is $\mu=$ $1.4 \pm 0.3$, which is consistent with the SM Higgs boson hypothesis $\mu=1$. Overall, the results in all channels (Fig. 13) are consistent with the SM Higgs boson hypothesis.

Conclusions and outlook. The high degree of statistical significance and simultaneous observation in multiple channels and data sets in this search 
Fig. 12. Confidence intervals comparing mass and signal strength for the $\mathrm{H} \rightarrow \gamma \gamma, \mathrm{H} \rightarrow \mathrm{ZZ} \rightarrow \ell \ell \ell$, and $\mathrm{H} \rightarrow \mathrm{WW} \rightarrow \ell v e v$ channels, including all systematic uncertainties. The markers indicate the maximum likelihood estimates $\left(\mu, m_{\mathrm{H}}\right)$ in the corresponding channels (the maximum likelihood estimates for $\mathrm{H} \rightarrow \mathrm{ZZ} \rightarrow$ elel and $\mathrm{H} \rightarrow \mathrm{WW} \rightarrow \ell v \ell v$ coincide). Collision energy $=$ $7 \mathrm{TeV}, \mathrm{L}=4.7$ to $4.8 \mathrm{fb}^{-1}$; collision energy $=8 \mathrm{TeV}$, $\mathrm{L}=5.8$ to $5.9 \mathrm{fb}^{-1}$.

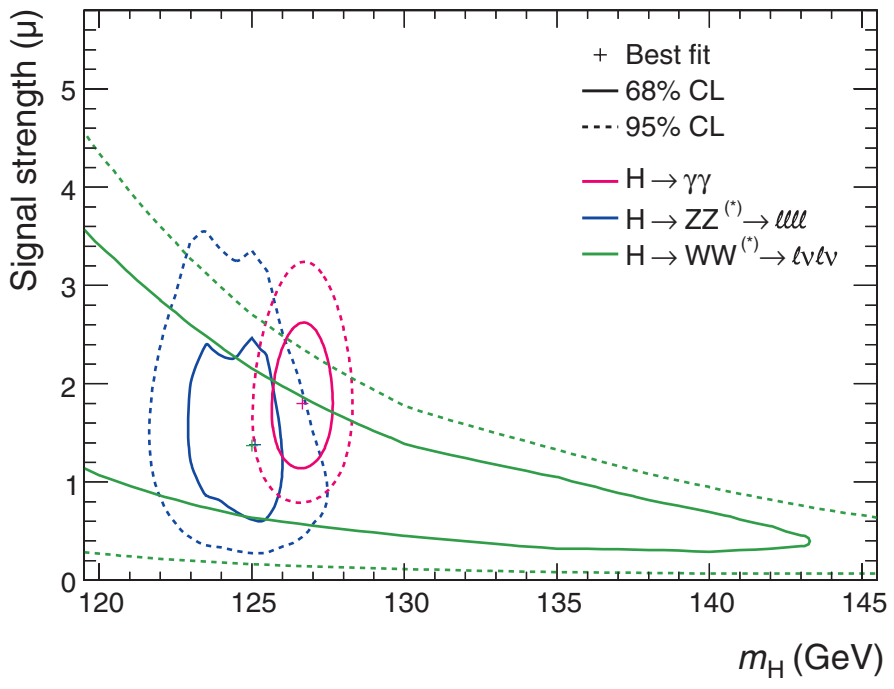

for decays to $b$ quarks and $\tau$ leptons are not yet sensitive enough to give conclusive results.

The energy density of the SM vacuum field is predicted to fill all of space. The nonzero vacuum energy density can be verified further by measuring the Higgs boson self-coupling, which can be accomplished by observing the production of multiple Higgs bosons. Because the expected production rate is small, this will be a challenge for the future high-intensity LHC program. General relativity normally associates a gravitational attraction to energy density. The impact of the energy density of the SM vacuum field on cosmology should be large. Because such effects are not observed, the relationship between the SM vacuum energy density and gravitation remains to be explored.

A relatively light Higgs boson suggests that new physical phenomena may exist at energies not far above the measured mass. Without new phenomena, quantum loop processes would drive the predicted Higgs boson mass far above the highest energy scale at which the SM is valid. For example, a theoretical model known as supersymmetry could provide a natural explanation for the light mass. Supersymmetry unifies matter and forces and for every known particle predicts a new "superpartner," some of which would enter into the quantum loops affecting the Higgs boson mass. The ATLAS and CMS experiments are actively searching for the first direct evidence of these superpartners. Various models, including supersymmetry, suggest that five distinct types of Higgs bosons exist. Therefore, another key issue is whether the observed boson is the only Higgs boson.

The groundbreaking discovery of a SM Higgslike boson may have identified the last missing piece of the SM as originally envisaged but is also an inspiration for further studies of the newly discovered boson, which might be a means to explore the physics that must lie beyond the SM. The LHC and its experiments are expected to address this new challenge in the coming years.
References and Notes

1. S. L. Glashow, Nucl. Phys. 22, 579 (1961).

2. S. Weinberg, Phys. Rev. Lett. 19, 1264 (1967).

3. A. Salam, in Elementary Particle Theory, N. Svartholm, Ed. (Almqvist and Wiksell, Stockholm, 1968) pp. 367-377.

4. G. 't Hooft, M. Veltman, Nud. Phys. B 44, 189 (1972).

5. F. Englert, R. Brout, Phys. Rev. Lett. 13, 321 (1964)

6. P. W. Higgs, Phys. Lett. 12, 132 (1964).

7. P. W. Higgs, Phys. Rev. Lett. 13, 508 (1964)

8. G. S. Guralnik, C. R. Hagen, T. W. B. Kibble, Phys. Rev. Lett. 13, 585 (1964).

9. P. W. Higgs, Phys. Rev. 145, 1156 (1966).

10. T. W. B. Kibble, Phys. Rev. 155, 1554 (1967).

11. ALEPH, CDF, DØ, DELPHI, L3, OPAL, SLD Collaborations, LEP Electroweak Working Group, Tevatron Electroweak Working Group, SLD electroweak and heavy flavour groups, http://arxiv.org/abs/1012.2367 (2010).

12. More recent results are available at http://lepewwg.web. cern.ch/LEPEWWG/plots/winter2012.

13. ALEPH, DELPHI, L3, OPAL, SLD Collaborations, LEP Electroweak Working Group, SLD Electroweak and Heavy Flavour Groups, Phys. Rep. 427, 256 (2006).

14. In the literature of particle physics, it is common to use natural units where the value of the velocity of light in vacuum is 1 , so that mass, momentum, and energy all have units of electron volts.

15. LEP Working Group for Higgs boson searches, ALEPH, DELPHI, L3, OPAL, Phys. Lett. B 565, 61 (2003).

16. CDF Collaboration, Dø Collaboration, Tevatron New Physics, Higgs Working Group, http://arxiv.org/abs/1207.0449 (2012).

17. L. Evans, P. Bryant, Eds., JINST 3, S08001 (2008).

18. ATLAS Collaboration, JINST 3, S08003 (2008).

19. CMS Collaboration, JINST 3, S08004 (2008).

20. ATLAS Collaboration, Phys. Rev. D 86, 032003 (2012).

21. CMS Collaboration, Phys. Lett. B 710, 26 (2012).

22. ATLAS Collaboration, Phys. Lett. B 716, 1 (2012).

23. CMS Collaboration, Phys. Lett. B 716, 30 (2012).

24. CMS Collaboration, Science 338, 1569 (2012).

25. M. Della Negra, P. Jenni, T. S. Virdee, Science 338, 1560 (2012).

26. LHC Higgs Cross Section Working Group, S. Dittmaier, C. Mariotti, G. Passarino, R. Tanaka, Eds., http://arxiv.org/ abs/1101.0593 (2011).

27. LHC Higgs Cross Section Working Group, S. Dittmaier, C. Mariotti, G. Passarino, R. Tanaka, Eds., http://arxiv.org/ abs/1201.3084 (2012)

28. ATLAS Collaboration, Eur. Phys. J. C 70, 823 (2010).

29. S. Agostinelli et al., Nucl. Instrum. Methods A506, 250 (2003).

30. The symbol $\ell$ stands for electron, $e$, or muon, $\mu$, for the decay modes examined in this article.

31. A. J. Barr, B. Gripaios, C. G. Lester, J. High Ener. Phys. 0907, 072 (2009).

32. T. Aaltonen et al., CDF Collaboration, DØ Collaboration, Phys. Rev. Lett. 109, 071804 (2012).

33. L. D. Landau, Dokl. Akad. Nawk. USSR 60, 207 (1948)

34. C. N. Yang, Phys. Rev. 77, 242 (1950).

Acknowledgments: These results would not have been possible without the outstanding performance of the LHC. We thank CERN and the entire LHC project team, including the operation, technical, and infrastructure groups and all the people who have contributed to the conception, design, and construction of this superb accelerator. We thank also the support staff at our institutions, without whose excellent contributions ATLAS could not have been successfully constructed or operated so efficiently, and all Worldwide LHC Computing Grid partners for their crucial computing support. We also thank all of the funding agencies of the many countries that contributed, over two decades, to the construction and the operation of the ATLAS experiment.

\section{Supplementary Materials}

www.sciencemag.org/cgi/content/ful//338/6114/1576/DC1 Complete Author List

10.1126/science. 1232005 


\section{Science}

\section{A Particle Consistent with the Higgs Boson Observed with the ATLAS Detector at the Large Hadron Collider}

The ATLAS Collaboration

Science 338 (6114), 1576-1582.

DOI: $10.1126 /$ science. 1232005

ARTICLE TOOLS

SUPPLEMENTARY

MATERIALS

RELATED

CONTENT

REFERENCES

PERMISSIONS http://science.sciencemag.org/content/338/6114/1576

http://science.sciencemag.org/content/suppl/2012/12/19/338.6114.1576.DC1

http://science.sciencemag.org/content/sci/338/6114/1524.full http://science.sciencemag.org/content/sci/338/6114/1558.full

This article cites 24 articles, 2 of which you can access for free http://science.sciencemag.org/content/338/6114/1576\#BIBL

http://www.sciencemag.org/help/reprints-and-permissions

Use of this article is subject to the Terms of Service

Science (print ISSN 0036-8075; online ISSN 1095-9203) is published by the American Association for the Advancement of Science, 1200 New York Avenue NW, Washington, DC 20005. 2017 (C) The Authors, some rights reserved; exclusive licensee American Association for the Advancement of Science. No claim to original U.S. Government Works. The title Science is a registered trademark of AAAS. 